\title{
Características morfológicas y cualitativas de variedades de cebolla en tres épocas de trasplante
}

\author{
Morphological and qualitative characteristics of onion varieties in three \\ transplanting dates
}

\author{
Hugo Alberto Zarza Silva ${ }^{1 *}$, Cipriano Ramón Enciso Garay ${ }^{2}$ y Fátima Natalia \\ González Ferreira ${ }^{2}$
}

${ }^{1}$ Centro de Investigación Hernando Bertoni, Instituto Paraguayo de Tecnología Agraria. Caacupé, Paraguay.
${ }^{2}$ Facultad de Ciencias Agrarias, Universidad Nacional de Asunción. San Lorenzo, Paraguay.
$\begin{array}{r}* \text { Autor para correspondencia (hugozarza@ @utlook.com) } \\ \text { Recibido: 19/12/2014; Aceptado: 06/04/2015. }\end{array}$

\section{RESUMEN}

Diferentes épocas de trasplante y sus efectos en las características morfológicas y cualitativas de variedades de cebolla se evaluaron en Caacupé, Paraguay. Las 17 variedades (Valencianita Precoz, Victoria, Catarina, Yellow Grano, Caeté, Bahía Periforme, Lola, Dourada, Rainha, Aurora, Cascata, Alvorada, Primavera, Red Creole, Montana, Salto Grande y Bella Dura), se trasplantaron en tres fechas diferentes, 24/05/12, 26/06/12 y 06/08/12. El diseño experimental fue de parcelas divididas (17 x 3), donde la parcela principal fueron las variedades y la parcela secundaria las épocas de trasplante, distribuidos en bloques completos al azar con tres repeticiones. Los datos fueron sometidos al análisis de varianza y los promedios comparados por la prueba de Tukey al $5 \%$ de probabilidad. Hubo interacción significativa para las variables evaluadas, lo cual demuestra que las variedades se comportaron en forma diferenciada en las épocas de trasplante. El mayor número de hojas, diámetro de bulbo, porcentaje de bulbos comerciales y diámetro del cuello se obtuvieron en la primera época, mientras que el mayor porcentaje de peso oreado en la tercera época $(88,33 \%)$. Red Creole presentó el mayor diámetro de bulbo en las tres épocas de trasplante. En la primera época también se destacaron para la misma variable Aurora y Victoria, en la segunda época Yellow Grano y en el última Aurora, Rainha, Dourada, Caeté, Yellow Grano, Catarina, Victoria, Bella Dura y Valencianita. La variedad Lola presentó el mayor porcentaje de bulbos comerciales $(98,42 \%)$ en la primera época, Yellow Grano $(96,70 \%)$ en la segunda época y Red Creole $(93,78 \%)$ en la última época de trasplante.

Palabras clave: Allium cepa L., épocas de trasplante, fotoperiodo, variedades.

\begin{abstract}
Different transplanting dates and their effects on the morphological and qualitative characteristics of onions varieties were evaluated in Caacupé, Paraguay in 2012. The 17 varieties (Valencianita Precoz, Victoria, Catarina, Yellow Grain, Caeté, Bahía Periforme, Lola, Dourada, Rainha, Aurora, Cascata, Alvorada, Primavera, Red Creole, Montana, Salto Grande and Bella Dura), were transplanted in three different dates, 24/05/12, 26/06/12 and $06 / 08 / 12$. The experimental design was a split-plot $(17 \times 3)$, where the main plots were the cultivars and subplots were the dates of transplantation, distributed in a randomized complete block design with three replications. Data were subjected to analysis of variance and means compared by Tukey test at $5 \%$ probability. There were significant interaction between onion varieties and transplanting dates, demonstrating that varieties behave differently on different transplanting dates. The highest average number of leaves per plant, bulb diameter, percentage of commercial bulbs and neck diameter were obtained in the first date, while the highest percentage of curate onion weight in the second date. Red Creole bulbs presented larger diameter in the three transplanting dates. In the first transplanting date Aurora and Victoria also showed higher bulb diameters, in the second date Yellow Grain and in the last period Aurora, Rainha, Dourada, Caete, Yellow Grain, Catarina, Victoria, Bella Dura and Valencianita. The Lola variety had the highest percentage of commercial bulbs (98.42\%) in the first date, Yellow Bean $(96.70 \%)$ in the second date and Red Creole $(93.78 \%)$ in the last date of transplantation.
\end{abstract}

Key words: Allium cepa L., transplanting dates, photoperiod, varieties. 


\section{INTRODUCCIÓN}

La cebolla (Allium cepa L.) es una especie hortícola que ocupa el segundo lugar en importancia económica mundial, siendo el mayor productor China con 15 millones de $\mathrm{t}$ año ${ }^{-1}$, mientras que Corea del Sur tiene la mejor productividad con $50 \mathrm{t} \mathrm{ha}^{-1}$. En Sudamérica, los mayores productores son Brasil y Argentina con 1.200 .000 y $650.000 \mathrm{t}^{\mathrm{año}}{ }^{-1}$ y rendimientos que oscilan de 33 a $15 \mathrm{t} \mathrm{ha}^{-1}$, respectivamente (FAO 2013).

En Paraguay, la superficie cultivada en el año 2013 fue de 796 ha, una producción de 4.925 t y rendimiento de $6,25 \mathrm{t}$ $\mathrm{ha}^{-1}$ (MAG/DCE 2013). La cosecha se concentra entre los meses de setiembre a noviembre y cubre el $33 \%$ de la demanda local (MAG/DC 2013). Entre las causas del bajo rendimiento y la concentración de la cosecha en solo tres meses, se encuentran la utilización de variedades no adaptadas a las condiciones locales y las épocas de siembra inoportunas.

Para cada región, la época de siembra y la variedad son los dos factores de manejo que diferencian la respuesta productiva en relación a los requerimientos en fotoperiodo y temperatura (Ansari 2007). Según Giaconi y Escaff (2004), las variedades de cebolla de acuerdo a las exigencias en fotoperiodo se clasifican en: de día corto (forman el bulbo cuando el largo del día excede 12 horas luz), de día intermedio (forman el bulbo cuando el largo del día excede 13 horas luz) y de día largo (forman el bulbo cuando el largo del día excede 14 horas luz). Una vez alcanzado el requerimiento fotoperiódico de la variedad, la temperatura incide en la tasa de crecimiento del bulbo (Arboleya 2005a).

El rango de temperatura para el crecimiento vegetativo es de 20 a $25^{\circ} \mathrm{C}$ y la óptima para la formación de bulbo oscila entre 25 y $30^{\circ} \mathrm{C}$ (Galmarini 1997), mientras que las superiores a $32^{\circ} \mathrm{C}$ pueden promover la bulbificación precoz (Melo y Churata - Masca 1988). En siembras muy tempranas las plantas emitirán tallo floral en lugar de formar bulbos $y$ en siembras tardías, las altas temperaturas estimulan la formación de bulbos cuando las plantas son pequeñas aun, razón por la cual se forman bulbos pequeños y de mala calidad, debido a la insuficiente formación de área foliar (Tesfay et al. 2011, Bosekeng y Coetzer 2010).

En el Paraguay debido a las condiciones de fotoperiodo reinantes se cultivan las variedades de día corto e intermedio con exigencias de 10 a 13 horas luz (Enciso
Garay y Román 2011), y ciclo de cuatro a seis meses desde la siembra hasta la cosecha. La siembra se realiza comúnmente entre los meses de abril a junio, sin embargo es necesario realizar trabajos de investigación que determinen las épocas óptimas de siembra para las nuevas variedades que se ofertan, de tal modo a ampliar el periodo de cosecha y mejorar la calidad de la producción.

Por lo mencionado, éste trabajo de investigación tiene como objetivo determinar los efectos de tres épocas de trasplante en las características agronómicas de 17 variedades de cebolla, en las condiciones del Departamento de Cordillera.

\section{MATERIALES Y MÉTODOS}

Este trabajo se realizó de febrero a diciembre del 2012, en el Centro de Investigación "Hernando Bertoni" (CIHB), Instituto Paraguayo de Tecnología Agraria (IPTA), ubicado en Caacupé, Departamento de Cordillera, Paraguay situado a $24^{\circ} 50^{\prime}$ latitud Sur, 56 $30^{\circ}$ longitud Oeste y altitud de $225 \mathrm{msnm}$. El clima del local está clasificado como subtropical con medias anuales de precipitación de $1.536 \mathrm{~mm}$ y temperatura de $22^{\circ} \mathrm{C}$. La evolución del fotoperiodo anual se presenta en la figura 1 y en la figura 2 la temperatura registrada en el año 2012.

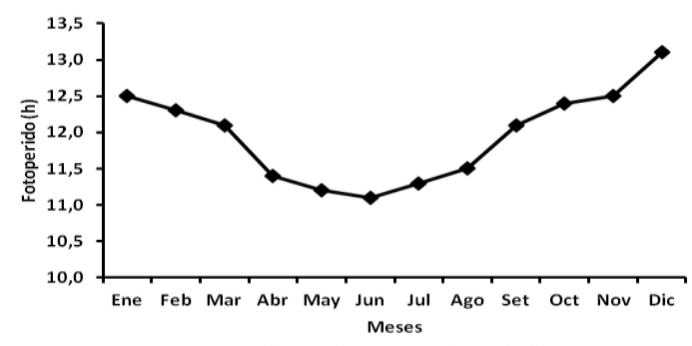

Figura 1. Fotoperiodo medio mensual en el año 2012. Datos proporcionados por la estación meteorológica del CIHB.

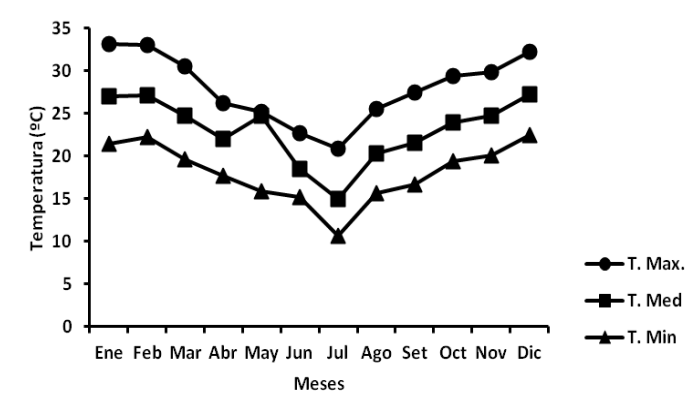

Figura 2. Temperatura mínima, máxima y media mensual en el año 2012. Datos proporcionados por la estación meteorológica del CIHB. 
Los suelos son originarios de arenisca, con predominancia de los Alfisoles y en un menor grado Ultisoles y Entisoles (López et al. 1995).

Los tratamientos estuvieron constituidos por la combinación de 17 variedades de cebolla (Valencianita Precoz, Victoria, Catarina, Yellow Grano, Caeté, Baía Periforme, Lola, Dourada, Rainha, Aurora, Cascata, Alvorada, Primavera, Red Creole, Montana, Salto Grande y Bella Dura), trasplantadas en tres épocas (24/05/12; 26/06/12 y 06/08/12). El diseño experimental fue de parcelas divididas distribuido en bloques completos al azar (BCA) con tres repeticiones, donde la parcela principal estuvo representada por las variedades y la parcela secundaria por las épocas de trasplante.

La producción de mudas se efectuó en almácigos, realizándose la siembra en las fechas 27/03/12; 27/04/12 y 04/06/12, para la primera, segunda y tercera época de trasplante.

Para el lugar definitivo, el suelo fue removido con un arado a discos y una rastra liviana, previa distribución del estiércol vacuno. La fertilización se realizó de acuerdo al análisis de suelo, el estiércol vacuno se distribuyó a razón de $10 \mathrm{t} \mathrm{ha}^{-1}$, además se aplicaron $40 \mathrm{~kg} \mathrm{ha}^{-1}$ de $\mathrm{K}_{2} \mathrm{O}$ y 100 $\mathrm{kg} \mathrm{ha}{ }^{-1}$ de $\mathrm{N}$, fraccionado en cuatro aplicaciones, la primera cinco días antes del trasplante; desde la segunda hasta la cuarta aplicación se realizaron a intervalos de 15 días, de la primera aplicación.

El trasplante se efectuó cuando las mudas presentaron cuatro hojas definitivas a una distancia de $30 \mathrm{~cm}$ entre hileras y $15 \mathrm{~cm}$ entre plantas. La unidad experimental tuvo 1,5 m de ancho y $3 \mathrm{~m}$ de longitud, con seis hileras del cultivo. El sistema de riego utilizado fue por aspersión.

Las malezas se controlaron de forma manual durante todo el ciclo. Luego del trasplante y posteriormente cada 15 días se aplicó una solución de insecticida compuesto por carbaryl $80 \%$ e imidacloprid $70 \%$ a razón de 30 g y 5 g por $20 \mathrm{~L}$ de agua, para evitar el ataque de Atta sexdens L. y Thrips tabaci Lind. Además se aplicó una mezcla de metalaxil $8 \%$ y mancozeb $64 \%$ con la misma frecuencia a razón de $50 \mathrm{~g}$ por $20 \mathrm{~L}$ de agua para prevenir el ataque de Alternaria porri.

La cosecha se efectuó en forma manual cuando el $80 \%$ de las plantas de cada tratamiento presentaron ablandamiento del cuello, el pseudotallo doblado y las hojas comenzaron a secarse. Luego de la cosecha los bulbos fueron llevados a un galpón donde permanecieron durante una semana para el oreado y curado.

Las variables medidas fueron número de hojas activas en el momento de la cosecha, diámetro del cuello en el momento de la cosecha, diámetro de bulbo, porcentaje de bulbos comerciales, porcentaje de peso oreado de bulbos respecto al peso fresco. Se consideró como bulbo comercial al que contuvo masa igual o superior a $50 \mathrm{~g}$. Los datos fueron sometidos al análisis de varianza y para la comparación de medias se utilizó la prueba de Tukey al $5 \%$ de probabilidad de error.

\section{RESULTADOS Y DISCUSIÓN}

\section{Número de hojas por planta}

El análisis estadístico detectó interacción significativa entre épocas de trasplante y variedades para el número de hojas (Tabla 1). Se verificó que las variedades Valenciana Precoz, Yellow Grano, Dourada, Aurora, Alvorada, Primavera, Red Creole y Salto Grande no difirieron estadísticamente en las tres épocas de trasplante. Por su parte Caeté, Victoria, Baia Periforme y Cascata produjeron mayor número de hojas en la primera época, difiriendo estadísticamente de las otras épocas. Bella Dura, Catarina, Lola y Rainha no presentaron diferencias entre la primera y tercera época. Montana fue la única variedad que no presentó diferencia estadística en el número de hojas entre la primera y segunda época.

Al comparar las variedades entre sí (Tabla 1) se verificó que en la primera época Cascata (9,20 hojas), produjo la mayor media, superando estadísticamente a Valencianita Precoz, Catarina, Yellow Grano, Dourada, Alvorada, Primavera, Red Creole y Salto Grande. En la segunda época Montana (7,46 hojas), produjo la mayor media, sin diferir de Primavera, Alvorada, Rainha, Lola, Yellow Grano, Catarina, Victoria, Bella Dura y Valencianita Precoz, pero fue superior estadísticamente a las demás. En la tercera época Caeté $(7,13$ hojas $)$ superó estadísticamente a Baia Periforme (4,73 hojas) y Alvorada (4,60 hojas).

Al comparar el número de hojas por planta de las variedades evaluadas en las tres épocas de trasplante se encontró que Caeté (7,31 hojas) promovió la mayor media, difiriendo de Valencianita Precoz, Victoria, Catarina, Yellow Grano, Dourada, Alvorada, Primavera y Red Creole. 
Tabla 1. Número de hojas por planta en el momento de la cosecha de 17 variedades de cebolla en tres épocas de trasplante. CIHB, Caacupé, 2012.

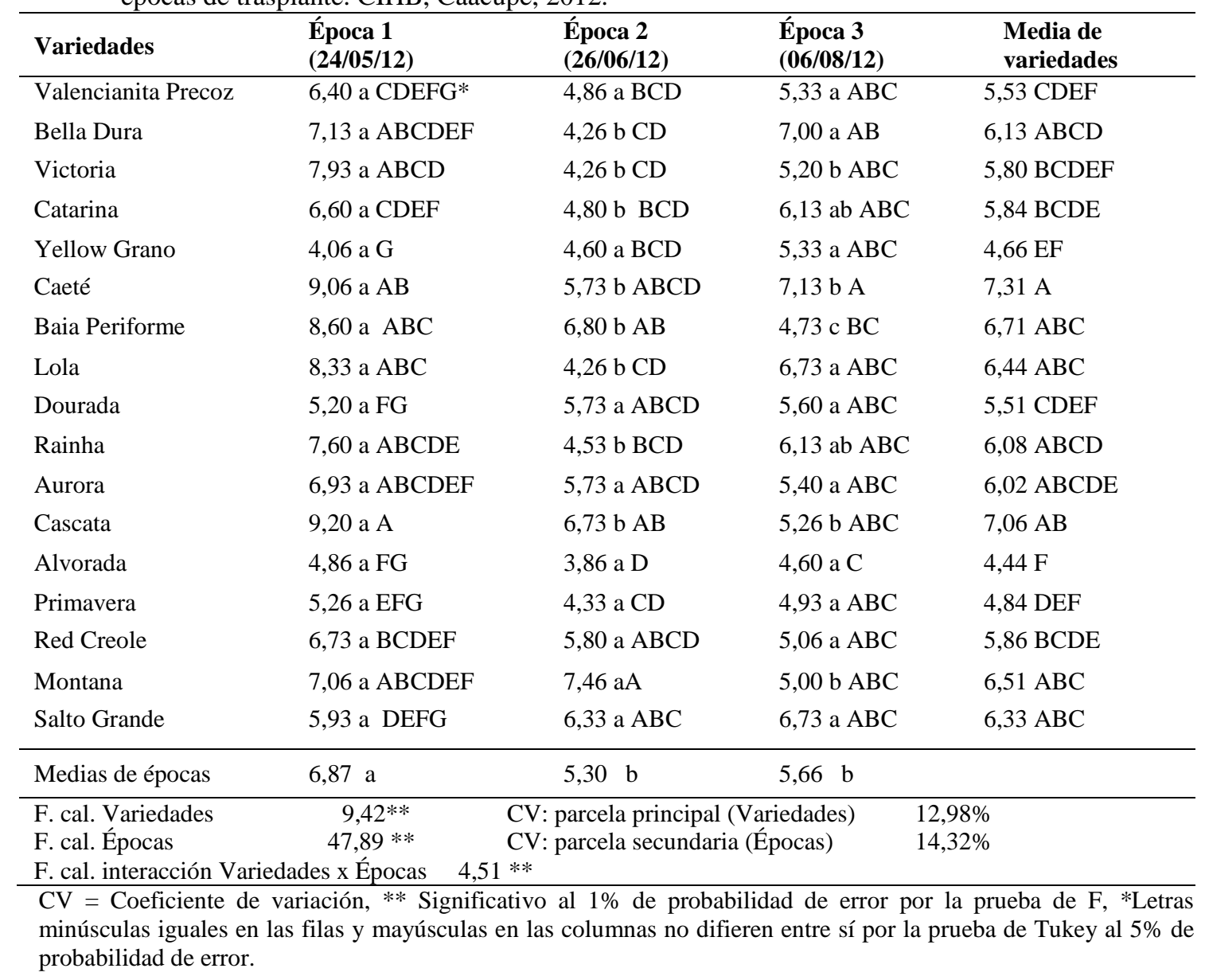

La variedad Baia Periforme presentó entre 8,60 y 4,73 hojas por planta, mientras que Red Creole entre 6,73 y 5,06 hojas por planta, que son inferiores a las medias de 9,63 y 9,83 hojas por planta reportado por Resende et al. (2007), para las mismas variedades en investigaciones realizadas en el Estado de Paraná, Brasil.

Comparando las medias de las diferentes variedades en cada época de trasplante, se verificó que en la primera época se obtuvo la mayor media (6,87 hojas) difiriendo estadísticamente de la segunda y tercera época de trasplante (Tabla 1). Este resultado coincide en cierta forma con lo presentado por Enciso Garay y Román (2011) quienes observaron que al atrasar la época de trasplante en tres variedades de cebolla, también se reduce el número de hojas por planta. Del mismo modo Ibrahim (2010) reportó diferencias significativas en el número de hojas por planta en la cebolla variedad Ex-Gidan Kwano trasplantada en cinco épocas diferentes. En coincidencia con los autores mencionados anteriormente Kandil et al.
(2013) y Bosenkeng y Coetzer (2010) encontraron que el trasplante realizado más temprano promueve la producción de mayor cantidad de hojas, posibilitando de esa forma una mayor actividad fotosintética de la planta y, por lo tanto, el aumento de la acumulación de materia seca en el bulbo, debido a las condiciones climáticas más favorables.

El número de hojas por planta que presenta la cebolla puede estar relacionado con una mayor actividad fotosintética de la planta y por tanto una mayor producción de fotoasimilados que pueden permitir un mayor desarrollo de los bulbos.

\section{Diámetro de bulbo}

En la tabla 2 se puede verificar que hubo interacción significativa entre los factores evaluados y también diferencias para los factores en forma separada. Valencianita Precoz, Victoria, Catarina, Baia Periforme, Lola, Rainha, Aurora, Alvorada, Primavera y Red Creole 
promovieron el mayor diámetro de bulbo en la primera época de trasplante, lo cual indica que para obtener bulbos de mayor tamaño, el trasplante de dichas variedades se debe realizar en mayo. Dourada, fue la única variedad que no presentó diferencias en las tres épocas de evaluación, lo cual indica que puede ser trasplantada en cualquiera de las épocas, sin que sea afectado el diámetro de sus bulbos. Las variedades Montana, Caeté y Bella Dura no difirieron sus medias en las dos primeras épocas de trasplante. Yellow Grano trasplantada en la segunda época presentó el mayor diámetro de bulbo, difiriendo de las medias obtenidas en la primera y segunda época, lo cual indica que para esta variedad la mejor época de trasplante fue el 26 de junio.
Respecto al comportamiento de las variedades (Tabla 2), se encontró que en la primera época Red Creole $(7,87 \mathrm{~cm})$ fue estadísticamente similar a Aurora $(7,10 \mathrm{~cm})$ y Victoria $(6,67 \mathrm{~cm})$, pero superior a las demás. En la segunda época nuevamente se destacó Red Creole $(6,89 \mathrm{~cm})$, que difirió de todas, salvo de Yellow Grano $(6,01 \mathrm{~cm})$. En la última época la mayor media fue para Victoria $(5,24 \mathrm{~cm})$, la cual fue diferente estadísticamente de Baia Periforme, Lola, Cascata, Primavera, Alvorada, Montana y Salto Grande. Comparando la media de las variedades en las tres épocas de trasplante, se observó que Red Creole $(6,45 \mathrm{~cm})$, difirió de las demás, salvo de Victoria $(5,68 \mathrm{~cm})$ y Aurora $(5,60 \mathrm{~cm})$. Las variedades con menor diámetro de bulbo fueron Montana (3,70 cm), Salto Grande $(4,04 \mathrm{~cm})$, Alvorada $(4,22 \mathrm{~cm})$ y Primavera $(4,58 \mathrm{~cm})$.

Tabla 2. Diámetro $(\mathrm{cm})$ de bulbos de 17 variedades de cebolla en tres épocas de trasplante. CIHB, Caacupé, 2012.

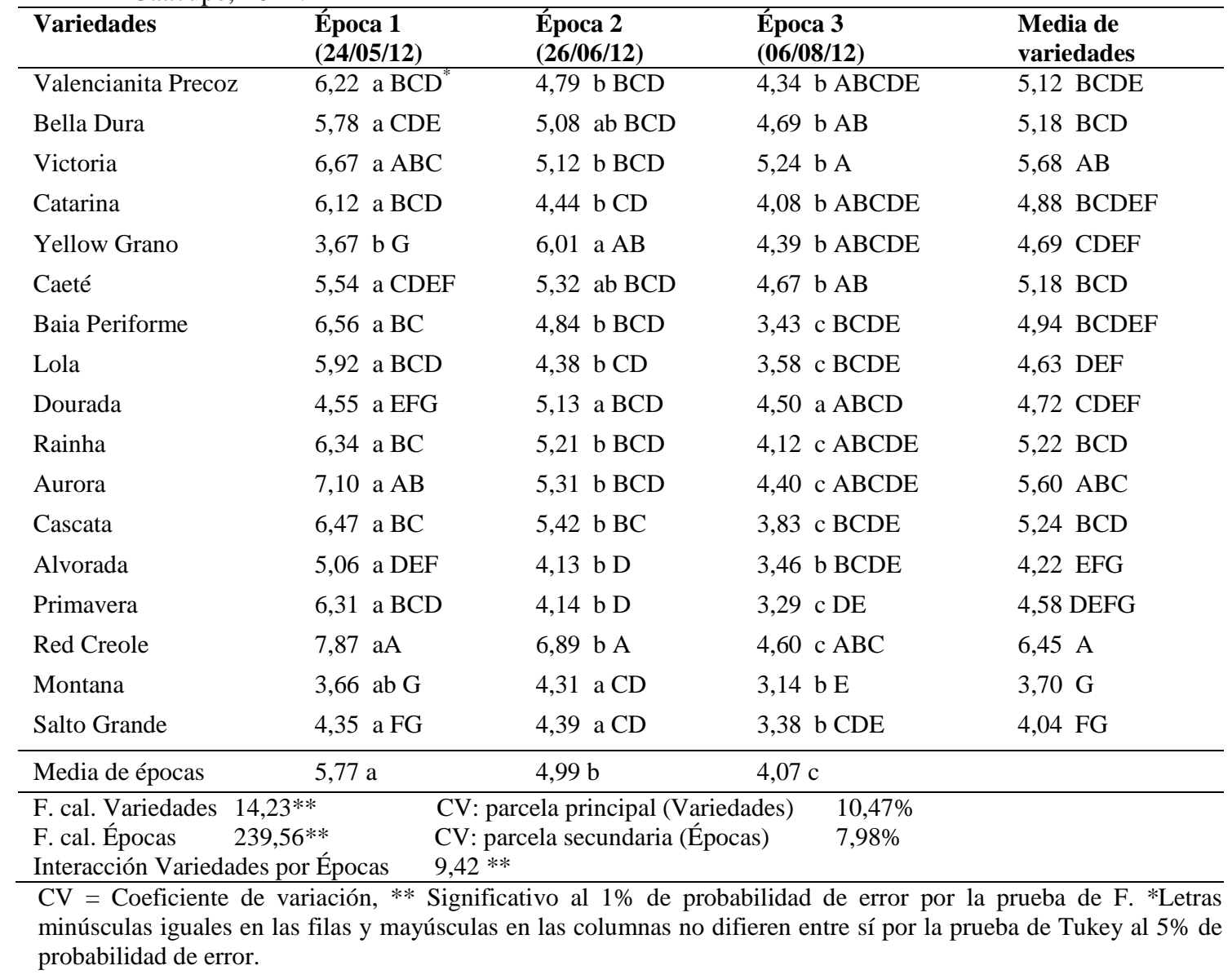

Comparando las medias de las épocas (Tabla 2), se constató que en la primera época $(5,77 \mathrm{~cm})$, se obtuvo la mayor media, difiriendo estadísticamente de la segunda $(4,99 \mathrm{~cm})$ que ocupó posición intermedia y, también de la tercera época $(4,07 \mathrm{~cm})$, que presentó la menor media, lo cual indica que al atrasar la época de trasplante a partir del 24 de mayo se reduce el tamaño de bulbos. Las diferencias encontradas en el tamaño de bulbos en función a las épocas de trasplante de las variedades de cebolla, son coincidentes con los reportados por Qasem (2006) y Bosekeng y Coetzer (2010) quienes en sus investigaciones con variedades de cebolla sembradas en diferentes épocas, también encontraron diferencias significativas en el diámetro de bulbo en función a las 
épocas de trasplante y a las variedades. Lancaster et al. (1996) en su investigación sobre el efecto de la temperatura y fotoperiodo en cebolla mediante diferentes épocas de siembra reportaron que al atrasar el periodo de siembra se reduce el número de hojas por planta y el diámetro de bulbo.

El rango del diámetro de bulbo de las variedades evaluadas en la primera época de trasplante varió de 3,67 cm (Yellow Grano) a 7,87 cm (Red Creole), en la segunda época de 4,13 cm (Alvorada) a 6,89 cm (Red Creole) y en la última época de $3,14 \mathrm{~cm}$ (Montana) a $5,24 \mathrm{~cm}$ (Victoria). Resende et al. (2002) evaluando siete genotipos de cebolla, entre ellas Baia Periforme registraron medias para la misma variable entre $5,35 \mathrm{~cm}$ a $7,38 \mathrm{~cm}$.

Arboleya (2005b) señala que para cada variedad existe una época adecuada de siembra, si la siembra es temprana puede haber floración prematura y si es tardía se afectará el rendimiento y tamaño de los bulbos, a causa de la reducción del periodo en almacigo e inicio tardío de bulbificación y, mayores temperaturas durante el crecimiento de bulbos. Del mismo modo Galmarini (1997) menciona que los requerimientos térmicos y de fotoperiodo varían entre cultivares $y$ es preciso determinarlos para cada zona de producción, a fin de elegir la época de siembra adecuada. Esto puede explicar los resultados de esta investigación, atendiendo que las mudas trasplantadas en la primera época tuvieron condiciones climáticas más favorables para la formación de bulbos, mientras que las trasplantadas en la segunda y tercera época (26/06 y 06/08) ya tuvieron temperaturas más elevadas y fotoperiodo más largo durante el periodo de formación de bulbos (Figura 1 y 2).

\section{Porcentaje de bulbos comerciales}

Se pudo observar que el porcentaje de bulbos comerciales fue influenciado tanto por las variedades, como por las épocas de trasplante, debido a que la interacción entre ambos fue significativa (Tabla 3). Se verificó que en la primera época de trasplante las variedades evaluadas a excepción de Yellow Grano, promovieron las mayores medias. En las tres épocas de evaluación Valencianita Precoz, Caeté y Red Creole, no presentaron diferencias, lo cual indica que en estas variedades la época de trasplante no tuvo incidencia en el porcentaje de bulbos comerciales, mientras que Victoria, Catarina, Baia Periforme, Lola, Dourada, Alvorada y Salto Grande, no difirieron en las dos primeras épocas de trasplante. En la última época, todas las variedades a excepción de Valencianita Precoz, Caeté, Cascata, Red Creole y Montana produjeron el menor porcentaje de bulbos comerciales.

Respecto al comportamiento de las variedades, en la primera época (Tabla 3), se verificó que Lola promovió la mayor media $(98,42 \%)$, difiriendo de Montana $(83,38 \%)$ y Cascata $(82,07 \%)$. En la segunda época Yellow Grano $(96,70 \%)$ superó estadísticamente a Cascata $(69,75 \%)$, Primavera $(79,55 \%)$, Montana $(80,32 \%)$ y Bella Dura $(81,84 \%)$. En la tercera época Red Creole $(93,78 \%)$, difirió estadísticamente de Primavera $(73,06 \%)$, Salto Grande $(58,19 \%)$ y Baia Periforme (sin bulbos comerciales). Al comparar el porcentaje de bulbos comerciales de las variedades evaluadas en las tres épocas de trasplante, Red Creole con media de 93,98\%, fue superior estadísticamente a Baia Periforme, Dourada, Cascata, Primavera, Montana y Salto Grande. Estos resultados son diferentes a lo reportado por Duarte et al. (2003) que en investigación realizada en Brasil con 18 genotipos de cebolla obtuvieron entre $76 \%$ y $95 \%$ de bulbos comerciales.

El mayor porcentaje de bulbos comerciales se obtuvo en la primera época de trasplante (Tabla 3) que fue de $92,47 \%$, difiriendo significativamente de la segunda $(87,35 \%)$ y tercera época de trasplante $(78,05 \%)$. En la última época se registró la menor media, difiriendo estadísticamente de las demás épocas, lo cual demuestra que al atrasar la época de trasplante el porcentaje de bulbos comerciales disminuye, debido probablemente a la mayor temperatura y aumento del fotoperiodo durante el periodo de bulbificación (Figura 1 y 2).

\section{Porcentaje de peso de bulbo oreado}

La interacción entre variedades y épocas de trasplante fue significativa para el porcentaje de peso de bulbo oreado (Tabla 4). La variedad Salto Grande promovió la mayor media en la primera época, difiriendo de las otras épocas. Las variedades Caeté, Dourada, Rainha, Aurora, Alvorada y Primavera no difirieron en las tres épocas de trasplante. Bella Dura, Victoria, Catarina, Yellow Grano, Baia Periforme, Lola y Cascata promovieron la mayor media en las dos últimas épocas de trasplante.

Comparando las variedades entre sí, en la primera época de trasplante, Montana $(97,17 \%)$ generó la mayor media, difiriendo de Salto Grande, Lola, Cascata. Rainha, Baia Periforme, Caeté, Yellow Grano, Catarina, Victoria, Bella Dura y Valencianita Precoz. 
Tabla 3. Porcentaje de bulbos comerciales con respecto al número total de bulbos cosechados de variedades de cebolla en tres épocas de trasplante. CIHB, Caacupé, 2012.

\begin{tabular}{|c|c|c|c|c|}
\hline Variedades & $\begin{array}{l}\text { Época 1 } \\
(24 / 05 / 12)\end{array}$ & $\begin{array}{l}\text { Época } 2 \\
(26 / 06 / 12)\end{array}$ & $\begin{array}{l}\text { Época 3 } \\
(06 / 08 / 12)\end{array}$ & $\begin{array}{l}\text { Media de } \\
\text { variedades }\end{array}$ \\
\hline Valencianita Precoz & 87,94 aABC* & 85,77 a $\mathrm{ABCD}$ & 86,59 a $\mathrm{ABC}$ & $86,77 \mathrm{ABC}$ \\
\hline Bella Dura & 96,45 a $\mathrm{A}$ & 81,84 b CD & 88,98 ab AB & 89,09 AB \\
\hline Victoria & 95,07 a $\mathrm{AB}$ & 95,87 a $\mathrm{AB}$ & 79,61 b BCD & $90,18 \mathrm{AB}$ \\
\hline Catarina & 95,00 a $\mathrm{AB}$ & 89,60 ab ABCD & 86,97 b ABC & $90,52 \mathrm{AB}$ \\
\hline Yellow Grano & 89,15 b ABC & 96,70 aA & 83,00 b ABCD & $89,61 \mathrm{AB}$ \\
\hline Caeté & 94,38 a $\mathrm{AB}$ & 87,94 a $\mathrm{ABCD}$ & 87,05 a $\mathrm{ABC}$ & $89,79 \mathrm{AB}$ \\
\hline Baia Periforme & 96,09 aA & 90,10 a $\mathrm{ABCD}$ & $0,00 \quad b F$ & $62,06 \mathrm{E}$ \\
\hline Lola & 98,42 aA & 93,78 a AB & 76,62 b $\quad C D$ & $89,61 \mathrm{AB}$ \\
\hline Dourada & 89,86 a $\mathrm{ABC}$ & 87,30 a $\mathrm{ABCD}$ & 79,33 b BCD & 85,49 BCD \\
\hline Rainha & $95,66 \mathrm{aA}$ & 87,22 b ABCD & 85,86 b ABC & $89,58 \mathrm{AB}$ \\
\hline Aurora & 93,57 a $\mathrm{ABC}$ & 84,56 b BCD & 88,47 ab $\mathrm{AB}$ & $88,86 \mathrm{AB}$ \\
\hline Cascata & 82,07 a C & 69,75 b E & 82,07 a $\mathrm{AB}$ & $80,38 \mathrm{CD}$ \\
\hline Alvorada & 94,00 a $\mathrm{AB}$ & 92,33 a $\mathrm{ABC}$ & 80,87 b BCD & $89,07 \mathrm{AB}$ \\
\hline Primavera & 98,09 aA & 79,55 b DE & 73,06 b D & $83,57 \mathrm{BCD}$ \\
\hline Red Creole & 93,41 a $\mathrm{ABC}$ & 94,75 a $\mathrm{AB}$ & 93,78 aA & 93,98 A \\
\hline Montana & 83,38 ab BC & 80,32 b DE & 89,21 a $\mathrm{AB}$ & $84,30 \mathrm{BCD}$ \\
\hline Salto Grande & 89,48 a $\mathrm{ABC}$ & 87,66 a $\mathrm{ABCD}$ & 58,19 b E & $78,44 \mathrm{D}$ \\
\hline Media de épocas & $92,47 \mathrm{a}$ & $87,35 \mathrm{~b}$ & $78,05 \mathrm{c}$ & \\
\hline F. cal. Variedades & $24,61 * *$ & $\mathrm{CV}$ : parcela principal (Variedades) & $5,14 \%$ & \\
\hline F. cal. Épocas & $186,33^{* *}$ & CV: parcela secundaria (Épocas) & $4,45 \%$ & \\
\hline Interacción Variedades & por Épocas & $39,30 * *$ & & \\
\hline
\end{tabular}

Tabla 4. Porcentaje de peso oreado con respecto al peso fresco de 17 variedades de cebolla en tres épocas de trasplante. CIHB, Caacupé, 2012.

\begin{tabular}{|c|c|c|c|c|}
\hline Variedades & $\begin{array}{l}\text { Época 1 } \\
(24 / 05 / 12)\end{array}$ & $\begin{array}{l}\text { Época } 2 \\
(26 / 06 / 12)\end{array}$ & $\begin{array}{l}\text { Época 3 } \\
(06 / 08 / 12)\end{array}$ & $\begin{array}{l}\text { Media de } \\
\text { variedades }\end{array}$ \\
\hline Valencianita Precoz & 78,41 b BCDE* & 85,42 ab AB & 94,98 a A & $86,27 \mathrm{~A}$ \\
\hline Bella Dura & 73,04 b CDE & 88,31 a $\mathrm{AB}$ & $93,01 \mathrm{aA}$ & $84,79 \mathrm{~A}$ \\
\hline Victoria & 67,76 b E & 97,55 a $\mathrm{A}$ & 93,19 aA & $86,17 \mathrm{~A}$ \\
\hline Catarina & 78,44 b BCDE & 87,78 ab AB & 93,04 a A & $86,42 \mathrm{~A}$ \\
\hline Yellow Grano & 76,55 b BCDE & 90,68 a $\mathrm{AB}$ & $93,01 \mathrm{aA}$ & $86,74 \mathrm{~A}$ \\
\hline Caeté & 78,98 a BCDE & 86,33 а $\mathrm{AB}$ & 88,58 a $\mathrm{A}$ & $84,63 \mathrm{~A}$ \\
\hline Baia Periforme & 72,84 b DE & 89,30 a $\mathrm{AB}$ & $93,26 \mathrm{aA}$ & $85,13 \mathrm{~A}$ \\
\hline Lola & $67,70 \mathrm{~b} \mathrm{E}$ & 90,95 a $\mathrm{AB}$ & $92,12 \mathrm{aA}$ & $83,59 \mathrm{~A}$ \\
\hline Dourada & 90,29 a $\mathrm{AB}$ & 85.52 a $\mathrm{AB}$ & 89,30 aA & $88,37 \mathrm{~A}$ \\
\hline Rainha & 81,94 a BCDE & 82,37 a B & 90,88 aA & $85,06 \mathrm{~A}$ \\
\hline Aurora & 83,05 a $\mathrm{ABCD}$ & 88,92 a $\mathrm{AB}$ & $91,90 \mathrm{aA}$ & $87,95 \mathrm{~A}$ \\
\hline Cascata & 73,41 b CDE & 94,04 a $\mathrm{AB}$ & 96,43 aA & $87,96 \mathrm{~A}$ \\
\hline Alvorada & 87,89 a $\mathrm{ABC}$ & 89,78 а $\mathrm{AB}$ & 89,95 aA & $89,21 \mathrm{~A}$ \\
\hline Primavera & 85,81 a $A B C D$ & 88,30 a $\mathrm{AB}$ & 93,27 aA & $89,13 \mathrm{~A}$ \\
\hline Red Creole & 83,99 b ABCD & 96,39 а $\mathrm{AB}$ & $91,53 \mathrm{ab} \mathrm{A}$ & $90,63 \mathrm{~A}$ \\
\hline Montana & 97,17 aA & 83,91 b AB & 93,82 ab A & $91,63 \mathrm{~A}$ \\
\hline Salto Grande & 78,06 a $\mathrm{BCDE}$ & 66,62 b C & 23,19 с B & $55,95 \mathrm{~B}$ \\
\hline Medias de épocas & 79,72 b & 87,77 a & \multicolumn{2}{|l|}{88,32 a } \\
\hline F. cal. Variedades 2 & $22,21 * *$ & al. (Variedades) & $5,88 \%$ & \\
\hline F. cal. Épocas & $43,76 * *$ & cundaria (Época & & \\
\hline \multicolumn{4}{|c|}{ Interacción Variedades por Épocas } & \\
\hline
\end{tabular}


En la segunda época Victoria $(97,55 \%)$ fue diferente estadísticamente a Rainha y Salto Grande. En la tercera época todas las variedades fueron estadísticamente similares entre sí, pero superiores a Salto Grande $(23,19 \%)$. Por otro lado, comparando las medias del porcentaje de bulbos comerciales de las variedades en las tres épocas (Tabla 4), se constató que Salto Grande $(55,95 \%)$ promovió la menor media, difiriendo de las demás variedades, las cuales a su vez, son iguales estadísticamente entre sí.

En cuanto al factor épocas (Tabla 4), el trasplante realizado el 26/06/12 y el 06/08/12 correspondientes a la segunda y tercera época con $87,77 \%$ y $88,32 \%$ de peso oreado, respecto al peso fresco de bulbos, promovieron la mayor media, superando estadísticamente a la media obtenida en la primera época de trasplante. El contenido de humedad es una característica importante relacionada al almacenaje de la cebolla. Bulbos más pequeños y cultivares con alto contenido de materia seca tienen mejor capacidad para almacenar (Toledo et al. 1984).

\section{Diámetro del cuello}

Hubo interacción significativa entre variedades y épocas de trasplante (Tabla 5) para diámetro del cuello. Las variedades Victoria, Catarina, Baia Periforme y Lola generaron las mayores medias en la primera época, difiriendo de las otras épocas de evaluación. Valencianita Precoz, Bella Dura, Yellow Grano, Alvorada y Primavera no presentaron diferencias entre la primera y tercera época de trasplante. Las variedades Dourada, Aurora, Montana y Salto Grande fueron estadísticamente similares en las tres épocas de trasplante. Red Creole, Cascata y Caeté fueron similares estadísticamente en las dos primeras épocas de evaluación.

Tabla 5. Diámetro de cuello $(\mathrm{cm})$ de 17 variedades de cebolla en tres épocas de trasplante. CIHB, Caacupé, 2012.

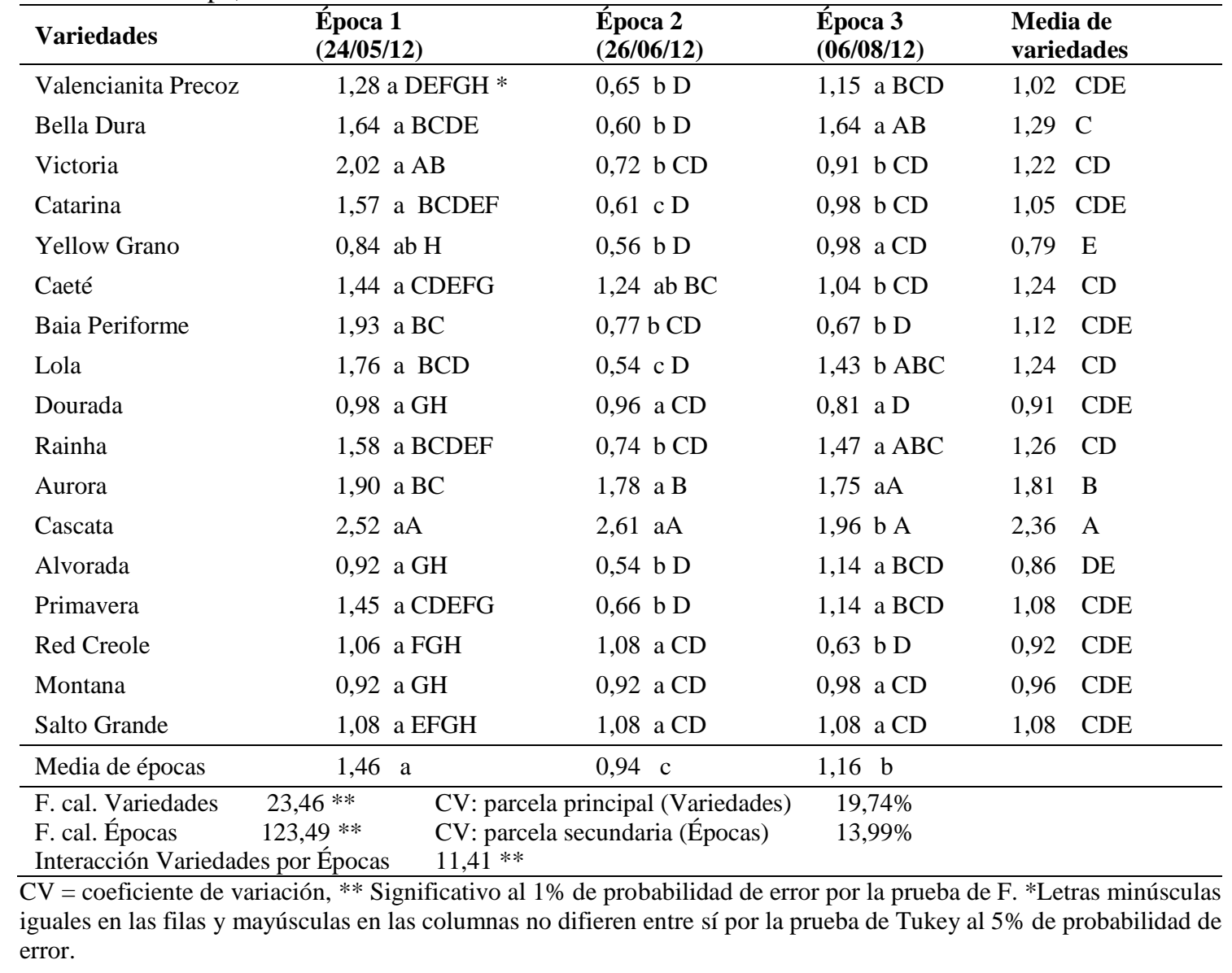

Al comparar las variedades, se constató que Cascata en la primera época de trasplante promovió la mayor media
$(2,52 \mathrm{~cm})$, difiriendo de todas, a excepción de Victoria, en la segunda época, con $2,61 \mathrm{~cm}$, fue superior a todas las 
variedades y en la tercera época $(1,96 \mathrm{~cm})$, no difirió de Aurora, Rainha y Lola, pero fue superior a las demás. Comparando las variedades en las tres épocas de trasplante, Cascata $(2,36 \mathrm{~cm})$ fue superior a todas, mientras que Yellow Grano $(0,79 \mathrm{~cm})$ generó la menor media.

Con relación a las medias obtenidas en las épocas (Tabla 5), se observó que la primera $(1,46 \mathrm{~cm})$ generó la mayor media difiriendo estadísticamente de las demás; la segunda época $(0,94 \mathrm{~cm})$ registró la menor media y fue diferente a la tercera época $(1,16 \mathrm{~cm})$, que ocupó posición intermedia.

La existencia de diferencias estadísticas entre épocas de trasplante y variedades en el diámetro del cuello son coincidentes con los resultados obtenidos por Bosekeng y Coetzer (2010) al evaluar cuatro variedades de cebolla en tres épocas de trasplante. Así mismo Resende et al. (2007) encontraron diferencias entre variedades de cebolla para la misma característica, reportando medias entre $1,64 \mathrm{~cm}$ y $2,10 \mathrm{~cm}$.

El diámetro del cuello es una variable considerada importante, cebollas con cuellos gruesos al momento de la cosecha serán un problema en la pos cosecha, el mismo debe estar cerrado y delgado para evitar podredumbres que afecten la calidad y el tiempo de almacenamiento (Carballo y Maeso 2005). Los mismos autores mencionan que para minimizar dicho problema se debe ajustar la fecha de siembra de las variedades. En la primera época de trasplante la variedad Yellow Grano presentó la menor media, difiriendo de Catarina, Victoria, Bella Dura, Caeté, Baia Periforme, Lola, Rainha, Aurora, Cascata y Primavera. En la segunda época de trasplante las variedades Lola y Alvorada $(0,54 \mathrm{~cm})$ generaron la menor media y estadísticamente fueron diferentes a Caeté, Aurora y Cascata. En la última época la variedad Red Creole presentó el menor diámetro del cuello, difiriendo de Cascata, Aurora, Rainha, Lola y Bella Dura.

Los resultados de esta investigación muestran que hubo diferencias estadísticas significativas para variedades y épocas de trasplante, en forma separada para todas las variables evaluadas. Así mismo, hubo interacción significativa entre los factores en estudio, lo cual indica la influencia de la temperatura y fotoperiodo en las características agronómicas de las variedades de cebolla, coincidiendo con trabajos realizados por Quartiero et al. (2014). Los mejores resultados obtenidos con el trasplante realizado en la primera época pueden ser debidos a que las plantas tuvieron temperaturas más adecuadas a los requerimientos del cultivo, sobre todo en los meses de junio, julio y agosto (Figura 2).

\section{CONCLUSIONES}

La primera época de trasplante promueve una mejor calidad concerniente a diámetro de bulbo y porcentaje de bulbos comerciales, razón por la cual es la más recomendada. El atraso en la época de trasplante reduce el número de hojas, porcentaje de bulbos comerciales, diámetro de bulbo y diámetro del cuello.

La variedad Red Creole presenta mayor diámetro de bulbo en las tres épocas de trasplante. En la primera época también se destacaron Aurora y Victoria; en la segunda Yellow Grano y en la tercera época Victoria, Catarina, Bella Dura, Valenciana Precoz, Yellow Grano, Caeté, Dourada, Rainha y Aurora.

La variedad Lola presenta el mayor porcentaje de bulbos comerciales en la primera época, Yellow Grano en la segunda y Red Creole en la tercera época.

El trasplante en la segunda y tercera época, promueven el mayor porcentaje de peso oreado de bulbos y el menor diámetro del cuello de las variedades evaluadas, lo cual puede permitir la obtención de bulbos con mayor potencial de almacenamiento.

\section{AGRADECIMIENTOS}

Al Gobierno de Corea por patrocinar esta investigación a través de Korea Project on International Agriculture (KOPIA).

\section{REFERENCIAS BIBLIOGRÁFICAS}

Ansari, NA. 2007. Effect of density, cultivars and sowing date on onion sets production. Asian J. Plant Sci. 6:1147-1150.

Arboleya J. 2005a. Actualidad de la producción de cebolla en Uruguay. In: Tecnología para la producción de cebolla. Arboleya, J. (ed.). Montevideo, UY, INIA. p. 1-15. (Boletín de Divulgación 88).

Arboleya, J. 2005b. Crecimiento y fisiología de la cebolla. In: Tecnología de producción de cebolla. Arboleya J. (ed). Montevideo, UY, INIA. p. 17-30. (Boletín de Divulgación 88). 
Bosekeng, G; Coetzer, GM. 2010. Response of Onion (Allium cepa L.) to sowing dates. African Journal of Agricultural Research 8(22): 2757-2764.

Carballo, S; Maeso, D. 2005. Manejo pos cosecha. In. Tecnología de producción de cebolla. Arboleya J. (ed). Montevideo, UY, INIA. p. 211-220.

Duarte, RLR; Veloso, MEC; Melo, FB; Sobrinho, CA; Ribeiro, VQ; Silva, PHS da. 2003. Produtividade de cultivares de cebola no Semi-Árido piauiense. Horticultura Brasileira 21(1): 34-36.

Enciso Garay, CR; Román; CA. 2011. Épocas de plantación y sus efectos sobre el rendimiento y calidad de bulbos de tres variedades de cebolla. Investigación Agraria 13(1):19-25.

FAO (Food and Agriculture Organization). 2013. Estimación de la producción mundial de cebolla. FAOSTAT. (en línea). Consultado 10 nov 2014. Disponible en: http://faostat3.fao.org/faostat-gateway/ go/to/home/E

Galmarini, CR. 1997. Manual del cultivo de la cebolla. Mendoza, AR, INTA. 128 p. (Manual No 16).

Giaconi, V; Escaff, M. 2004. Cultivo de hortalizas. 15 ed. Santiago, CL, Universitaria. 337 p.

Ibrahim, ND. 2010. Growth and yield of Onion (Allium cepa L.) in Sokoto, Nigeria Agric. Biol. J. N. Am. 1(4): 556-564.

Kandil, AE. Sharief, AE; Fathalla, HF.2013. Effects of transplanting dates of some onions cultivars on vegetative growth, yield and its quality. ESci Journal of Crop Production 2(3): 72-82.

Lancaster, JE; Triggs, CM; Ruiter, JM de; Gandar, PW. 1996. Bulbing in onions: photoperiod and temperature requeriments and prediction of bulb size and maturity. Annals of Botany 78:423 - 430.

López, O, González, E, Llamas, PA de, Molinas, AS, Franco, ES, García, S, Rios, EO. 1995. Estudio de reconocimiento de suelos, capacidad de uso de la tierra y propuesta de ordenamiento territorial preliminar de la región oriental del Paraguay. Asunción. PY. MAG. 246 p.
Qasem, JR. 2006. Response of onion (Allium cepa L.) plants to fertilizers, weed competition duration, and planting times in the central Jordan Valley. Weed Biology and Management. 6:212 - 220.

Quartiero, A; Faria, MV; Resende, JTV; Figueiredo, AST; Camargo, LKP; Santos, RL; Kobori, RF. 2014. Desempenho agronômico, heterose e estabilidade fenotípica de genótipos de cebola. Horticultura Brasileira 32(3): 259-266.

MAG (Ministerio de Agricultura y Ganadería, PY)/DCEA (Dirección de Censos y Estadísticas Agropecuarias). 2013. Síntesis Estadísticas: producción agropecuaria 2012/2013. San Lorenzo, PY. 47 p.

MAG (Ministerio de Agricultura y Ganadería, PY)/DC (Dirección de Comercialización). 2013. Ingresos y Precios Frutihortícolas en DAMA. (en línea). Consultado 20 nov 2014. Disponible en: ttp://www.mag.gov.py/dc/INGRESO\%20FRUTIHOR TICOLA\%20EN\%20DAMA/Cebolla.pdf

Melo, PC de; Churata-Masca, GCM. 1.988. Sistemas de produção, cultivares e o seu desenvolvimento para as condições brasileiras. In: III Seminario Nacional de Cebola. Jaboticabal. BR. FUNEP. p. $27-61$.

Resende, GM de; Chagas, SJR; Pereira, LV. 2002. Características produtivas de cultivares de cebola no Sul de Minas Gerais. (en línea) Horticultura Brasileira (20):2. Suplemento 2. Consultado 21 nov 2014. Disponible en: ttp://ainfo.cnptia.embrapa.br/digital/ bitstream/CPATSA/24831/1/OPB757.pdf

Resende, JTV; Pires, DB; Camargo, LKP; Marchese, A. 2007. Desempenho produtivo de cultivares de cebola em Guarapuava, Paraná. Ambiência 3(2):194-199.

Tesfay, SZ; Bertling I; Odindo AO; Greenfield, PL; Workneh, TS. 2011. Growth responses of tropical onion cultivars to photoperiod and temperature based on growing degree days. African Journal of Biotechnology 10(71): 15.875-15.882.

Toledo, J., M. Sherman, and D.J. Huber. 1984. Some effects of cultivar, bulb size and preharvest treatments on storage characteristics of North Florida onions. Proc. Florida State. Hort. Soc. 97:106-108. Disponible en: http://fshs.org/proceedings-o/1984-vol97/106-108.pdf 